\title{
Promiscuous Chemokine Antagonist (BKT130) Suppresses Laser-Induced Choroidal Neovascularization by Inhibition of Monocyte Recruitment
}

\author{
Shira Hagbi-Levi, ${ }^{1}$ Michal Abraham, ${ }^{2}$ Liran Tiosano, ${ }^{1}$ Batya Rinsky, ${ }^{1}$ Michelle Grunin, ${ }^{1}$ \\ Orly Eizenberg, ${ }^{2}$ Amnon Peled, ${ }^{2,3}$ and Itay Chowers ${ }^{1}{ }^{1}$ \\ ${ }^{1}$ Department of Ophthalmology, Hadassah-Hebrew University Medical Center, Jerusalem 91120, P.O.B 12000, Israel \\ ${ }^{2}$ Biokine Therapeutics Ltd., Science Park, Ness Ziona, Israel \\ ${ }^{3}$ Goldyne Savad Institute of Gene Therapy, Hadassah-Hebrew University Medical Center, Jerusalem 91120, P.O.B 12000, Israel \\ Correspondence should be addressed to Itay Chowers; chowers@hadassah.org.il
}

Received 7 January 2019; Revised 5 June 2019; Accepted 10 July 2019; Published 5 August 2019

Guest Editor: Heather Medbury

Copyright (c) 2019 Shira Hagbi-Levi et al. This is an open access article distributed under the Creative Commons Attribution License, which permits unrestricted use, distribution, and reproduction in any medium, provided the original work is properly cited.

\begin{abstract}
Background. Age-related macular degeneration (AMD), the most common cause of blindness in the developed world, usually affects individuals older than 60 years of age. The majority of visual loss in this disease is attributable to the development of choroidal neovascularization (CNV). Mononuclear phagocytes, including monocytes and their tissue descendants, macrophages, have long been implicated in the pathogenesis of neovascular AMD (nvAMD). Current therapies for nvAMD are based on targeting vascular endothelial growth factor (VEGF). This study is aimed at assessing if perturbation of chemokine signaling and mononuclear cell recruitment may serve as novel complementary therapeutic targets for nvAMD. Methods. A promiscuous chemokine antagonist (BKT130), aflibercept treatment, or combined BKT130+aflibercept treatment was tested in an in vivo laser-induced model of choroidal neovascularization (LI-CNV) and in an ex vivo choroidal sprouting assay (CSA). Quantification of CD11b+ cell in the CNV area was performed, and mRNA levels of genes implicated in CNV growth were measured in the retina and RPE-choroid. Results. BKT130 reduced the CNV area and recruitment of CD11b+cells by $30-35 \%$. No effect of BKT130 on macrophages' proangiogenic phenotype was demonstrated ex vivo, but a lower VEGFA and CCR2 expression was found in the RPE-choroid and a lower expression of TNF $\alpha$ and NOS1 was found in both RPE-choroid and retinal tissues in the LI-CNV model under treatment with BKT130. Conclusions. Targeting monocyte recruitment via perturbation of chemokine signaling can reduce the size of experimental $\mathrm{CNV}$ and should be evaluated as a potential novel therapeutic modality for nvAMD.
\end{abstract}

\section{Introduction}

Dysregulation of the complement and systemic immune systems has been associated with the pathogenesis of agerelated macular degeneration (AMD). Genetic, histological, and biochemical studies have associated the alternative complement pathway with the disease [1-8]. Lymphocytes, mononuclear cells, and particularly monocytes and macrophages were also implicated in AMD [3, 9-23]. In fact, infiltration of monocytes to the retina was found to be essential for the development of choroidal neovascularization (CNV)
$[18,24]$. Increased numbers of CD56+ $\mathrm{T}$ cells have been detected in the blood of AMD patients when compared to age-matched controls [25], and the interaction of T cells and M1 macrophages was reported during the stages of AMD [23]. Once recruited to the eye, monocytes differentiate to macrophages that can exert a proangiogenic effect in the context of neovascular AMD (nvAMD), an effect that may be exacerbated in aging $[17,18,22,26-31]$. Activated macrophages from nvAMD patients might exert a more significant proangiogenic effect compared with macrophages from agematched controls [22]. Several macrophage-derived cytokines, 
in addition to VEGF, can mediate CNV growth $[32,33]$. Accordingly, perturbation of monocyte recruitment and/or function may potentially result in suppression of CNV growth that may potentially complement anti-VEGF-based therapies.

Chemokines and their receptors play a critical role in the progression of autoimmune and inflammatory diseases such as AMD. Multiple chemokines were found to be involved in the development of this pathology [34-38]. For example, a transcriptome-wide analysis of the AMD donor retinas suggested that CCL2, IP-10, MIG, and I-TAC are upregulated in all forms of the disease [39]. CXCR3 is one of the mammalian chemokine receptors, promoting chemotaxis and cell proliferation. This receptor binds to three major chemokine ligands: IP-10, MIG, and I-TAC. CXCR3 expression and IP-10 were elevated in the RPE-choroid fractions of the laser-induced CNV eyes compared with nontreated fellow eyes [40]. Our group has reported an increased expression of other chemokine receptors, namely, CCR1 and CCR2, in the CD14+CD16+ subset of monocytes from neovascular AMD (nvAMD) patients [41]. CCR2 is a major chemokine receptor that is also potentially involved in macrophage activation and recruitment in AMD [32]. In accordance with the high levels of MCP-1, the ligand for CCR2 was detected in the aqueous humor of patients with AMD $[42,43]$, and macrophages have been found in the vicinity of drusen areas of retinal pigment epithelium (RPE) atrophy, Bruch's membrane rupture, and choroidal neovascularization $(\mathrm{CNV})$ in histological sections from AMD eyes [44-49].

Targeting a single chemokine, or its receptor, in an attempt to reduce macrophage recruitment to the retina was contemplated as a potential treatment for AMD. This approach is limited by the redundancy of the chemokine signaling system and by the nonexclusive nature of ligandreceptor interactions which characterizes it [50-52]. Here, we suggest an alternative approach involving antagonizing multiple chemokine signaling pathways simultaneously. Accordingly, a recent study demonstrated the efficiency of a broad-spectrum chemokine inhibitor (NR58-3.14.3) in modulating macrophage-mediated inflammation in light-induced retina injury [53].

BKT130 is a novel promiscuous chemokine-binding peptibody which has the ability to bind and inhibit multiple inflammatory chemokines, such as CCL2 (ligand for CCR2), CCL5 (binding CCR5), IP-10, MIG, and I-TAC (binding to CXCR3) [54]. This novel peptibody was already proven to have a therapeutic effect in autoimmune and inflammatory pathologies by inhibition of the recruitment of immune cells, inflammation, and disease progression in rodent models for rheumatoid arthritis (RA) and multiple sclerosis (MS) [54]. BKT130 was also found to inhibit melanoma and pancreatic tumor cell growth in mice [54]. In this study, we assessed the effect of this chemokine antagonist in a rodent model for laser injury-induced CNV and in complementary in vitro experiments.

\section{Materials and Methods}

2.1. Laser-Induced Model of CNV (LI-CNV) and Experimental Groups. LI-CNV was generated in adult Long-Evans rats
(8-12 weeks old). Animals were treated in accordance with the guidelines of the Association for Research in Vision and Ophthalmology (ARVO). Experiments were conducted with the approval of the institutional animal care ethics committee. Before each procedure, rats were anesthetized by intraperitoneal injections of a mixture of $85 \%$ ketamine (Bedford Laboratories, Bedford, $\mathrm{OH}$ ) and 15\% xylazine (VMD, Arendonk, Belgium). Local anesthesia using oxybuprocaine HCL 0.4\% (Localin) drops (Fisher Pharmaceuticals, Tel-Aviv, Israel) was applied to each eye 10 minutes before intravitreal injections or laser photocoagulation.

Laser burns (5-7 burns per eye) were generated as previously described [55]. Intravitreal injections were performed using a PLI-100 Pico-Injector (Medical System Corp., Greenvale, NY) as we have previously described [22]. Intravitreal injections of either $4 \mu \mathrm{l}$ of $5 \mathrm{mg} / \mathrm{ml}$ BKT130 (Biokine, Ness Ziona, Israel) ( $n=9$ eyes), $1 \mu \mathrm{l}$ of $40 \mathrm{mg} / \mathrm{ml}$ aflibercept (Bayer Pharma AG, Berlin, Germany) $(n=8)$, a combination of $4 \mu \mathrm{l}$ BKT130 and $1 \mu \mathrm{l}$ of aflibercept $(n=8)$, or $4 \mu \mathrm{l}$ of PBS solution $(n=10)$ were provided. BKT130 dosage was according to a previous study which tested dose response and kinetic analysis in vivo [54], and aflibercept dosage was according to that used in human eyes which was adjusted according to the size of the rat eye. All intravitreal injections were performed at the time of the laser burn injury and 5 days later. Antibiotic ointment (5\% Synthomycine) was applied after the injection. RPEchoroid flat mounts were dissected and processed for isolectin staining 10 days following the laser injury as we have previously described [22]. The contralateral retina of the same rat was homogenized and frozen at - 80 for RNA extraction using TRI-Reagent (Sigma-Aldrich, Munich, Germany).

2.2. CNV Quantification. RPE-choroid flat mounts were fixated for one hour in 4\% PFA and suspended overnight in isolectin solution (GS-Ib4 Alexa 594 staining solution, Molecular Probes, Eugene, Oregon) containing $200 \mathrm{mM}$ $\mathrm{NaN}_{3}$ and $1 \mathrm{mM} \mathrm{CaCl}$. Flat mounts were then washed 6 times for 20 minutes in PBS and embedded on a slide with a mounting medium. Isolectin images of the RPEchoroid flat mounts were viewed using a fluorescent microscope (Olympus BX41, Tokyo, Japan). Background was controlled by setting the exposure parameters as such so that they provided no detectable signal for the control nonimmune serum-stained rat flat mount. These same parameters were maintained while capturing all images from the test sections. Images were photographed with an Olympus DP70 digital camera.

The CNV area around each laser injury was measured using the ImageJ software [56]. The optic disc was removed to avoid autofluorescence from background counts. The scale was set to translate pixels into $\mathrm{mm}^{2}$, threshold was set on an unstained negative control, and these settings were used as background for all images. In order to calculate the average area of each CNV, we calculated the stained area of particles above the size of 60 pixels and divided it by the number of laser burns in the eye. The average CNV area of each eye was then calculated, and the mean CNV area of the four groups was compared. 
2.3. Immunohistochemistry. Immunohistochemistry of the fixed retinas was performed for mononuclear phagocyte $(\mathrm{CD} 11 \mathrm{~b}+)$ cell count. In brief, mouse monoclonal antiCD11b (Abcam, ab78457, 1:100) was used as the primary antibody for the rat mononuclear phagocytes. A donkey anti-mouse (Abcam, ab150110, 1:100) was used as the secondary antibody. Retinal flat mounts were first permeabilized and blocked for 3 hours at room temperature (RT) with blocking solution containing $0.1 \%$ Triton-X, $10 \%$ Normal Donkey Serum (NDS, Millipore S30, Temecula, CA, USA), $3 \%$ albumin bovine (BSA; Amresco Inc., Solon, OH, USA) in PBS. Primary antibody was added overnight in $4^{\circ} \mathrm{C}$ on shaker. Samples were then washed for 20 minutes six times in PBS at RT, and secondary antibodies were added for 2 hours on a shaker at RT. Samples were placed on slides with mounting medium after $4^{\prime}, 6^{\prime}$-diamidino-2-phénylindole (DAPI) (Enzo LifeScience Exeter, UK) staining, for cell nucleus identification. Flat mounts of eyes with LI-CNV and without primary antibodies served as negative controls, which defined our background for the microscopy.

Immunofluorescence analysis was performed using a Zeiss LSM 710 confocal laser scanning system (Carl Zeiss MicroImaging $\mathrm{GmbH}$, Jena, Germany) with 25X oil objective and a tile scan. Background was controlled by setting the exposure parameters as described above. These same parameters were maintained while capturing images from the test sections. CD11b+ cells which were found in the laser injury site, at the sub retinal space, were counted by a masked observer, using ImageJ software. The perimeter of the laser injury site was determined based on the absence of nearby photoreceptor cells as identified via DAPI staining, surrounding the laser injury (Figure 1(a)). Results are presented as the mean number of cells per laser-treated area of each experimental group \pm SEM.

2.4. Quantitative Real-Time PCR (QPCR). Total RNA was extracted from the flash-frozen retinas using TRI Reagent (Sigma-Aldrich), according to the manufacturer's instructions, and treated with DNase (TURBO DNA-free, Ambion, Austin, TX). Reverse transcriptase polymerase chain reaction was performed using the High Capacity cDNA Reverse Transcription Kits (Applied Biosystems, Foster City, CA) and anchored oligo dT primers on $1 \mu \mathrm{g}$ total RNA in a volume of $20 \mu \mathrm{l}$.

Quantitative real-time PCR (QPCR) was performed using the SYBR Green technique to measure mRNA levels of genes involved in angiogenesis, inflammation, mononuclear cell marker, macrophage polarization, and monocyte recruitment. Oligonucleotide primers for genes of interest [CCL2, CCR2, CCL5, VEGFA, IL1 $\beta$, TNF $\alpha$, NAP-2, MIP-2, CD11b, CD163, MRC1 (CD206), and N0S1] and for an endogenous control gene ( $\beta$-actin) were designed for QPCR using Primer-Blast (https://www.ncbi.nlm.nih.gov/tools/ primer-blast/). These genes were selected as they are related with chemokine signaling or with proangiogenic function of macrophages. All primers were purchased from SigmaAldrich (primer sequences are presented in Supplement Table 1). Measurement of the mRNA levels was performed on the retinas and RPE-choroid tissues, separately, 10 days
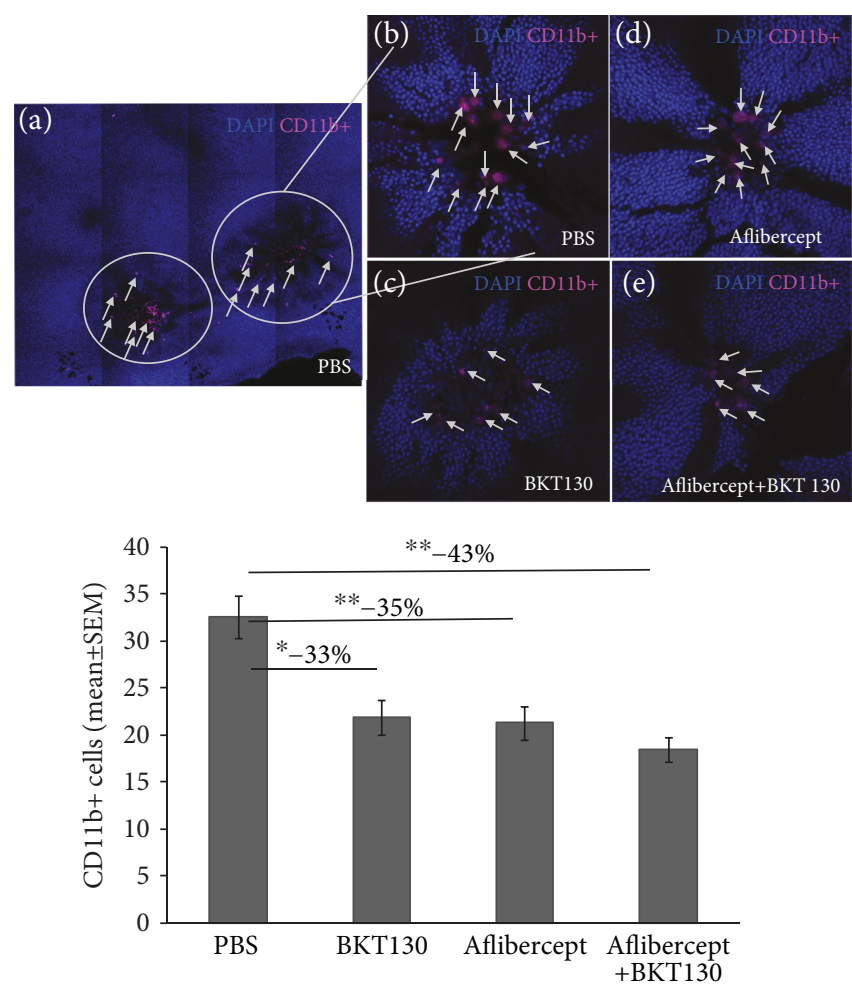

(f)

Figure 1: BKT130 reduces CD11b+ cell migration to the lasertreated area. Rat retinal flat mounts were prepared 10 days following laser injury and intravitreal injections. Flat mounts were observed using a confocal laser scanning system. CD11b-positive cells were observed in the center of the laser-treated areas (a). Each laser-treated area was observed in a 40x lens, and the macrophages (magenta) were counted $(b-e)$. The eyes injected with BKT130, aflibercept, or BKT130+aflibercept demonstrated less CD11b-positive cells ((d) and (e), respectively) compared with PBS-injected eyes (b). A comparison between the amounts of cells found in laser-treated areas in each group is provided in (f). The $Y$-axis presents the mean $( \pm \mathrm{SEM})$ number of $\mathrm{CD} 11 \mathrm{~b}+$ cells found in each laser-treated area, in either BKT130 (number of laser burns $=19)$, aflibercept $(n=25$ laser injury areas), BKT130+aflibercept $(n=41)$, or control PBS-injected group $(n=24) .{ }^{*} P<0.05$ and ${ }^{* *} P<0.005$.

after injections, in each experimental group ( $n=9$ eye samples in each group: PBS, BKT130, aflibercept, and, BKT130+aflibercept). Measurement of $\beta$-actin mRNA levels served as endogenous controls. All reactions were carried out in triplicate, using 384-well plates, at a total volume of $10 \mu \mathrm{l}$. Wells contained $20 \mathrm{ng}$ (for CCL2, CCR2, CCL5, $M R C 1, C D 163$, VEGFA, CD11b, NOS1, and IL1 $\beta$ ) or $100 \mathrm{ng}$ (for TNF $\alpha, N A P-2$, and MIP-2) cDNA template, $5 \mu \mathrm{l}$ of SYBR Green FastMix (Quanta Biosciences), and $0.5 \mu \mathrm{l}$ forward and reverse primers $(10 \mathrm{mM})$ for each gene. Signal amplification was measured throughout 38 cycles of $60^{\circ} \mathrm{C}$ for 20 seconds, followed by $95^{\circ} \mathrm{C}$ for 20 seconds. To confirm the amplification of a specific cDNA, the dissociation temperature was examined and compared with the calculated melting temperature for each amplified product. The amplified products were also examined by 
agarose gel electrophoresis. Fluorescent signals were measured by the CFX384, C1000 touch thermal cycler (Bio-Rad) and analyzed using the spreadsheet software (Excel; Microsoft, Redmond, WA). Expression levels of each gene were compared across the samples by using the expression levels of the endogenous control according to the standard $2^{(-\Delta \Delta \mathrm{CT})}$ calculation [57], giving results as relative quantification and fold change \pm standard error of the mean (SEM).

2.5. Choroid Sprouting Assay (CSA). Blood samples (20 ml) were collected from 6 nvAMD patients ( 3 females, 3 males, mean age \pm SEM: $70.8 \pm 2.3$ years, range: $64-81)$ in EDTA tubes (BD Bioscience). Patients were recruited from the retina clinic of the Department of Ophthalmology at the Hadassah-Hebrew University Medical Center. The criteria for the inclusion of nvAMD patients included the following: age over 55 years, diagnosis of AMD according to the AREDS criteria [58], and diagnosis of CNV according to fluorescein angiogram and optical coherence tomography. All patients signed an informed consent form, and the study was approved by the institutional ethics committee (see Ethical Approval). Monocytes were isolated from the whole blood, differentiated into macrophages (M0), and activated into M1- and M2a-like phenotypes, as we and others have previously described $[22,59,60]$.

An ex vivo angiogenesis assay was performed as previously described $[22,61]$, to evaluate the effect of BKT130 on the macrophages' proangiogenic phenotype. Briefly, the supernatant from human-activated and human-polarized macrophages that were treated with $50 \mu \mathrm{g} / \mathrm{ml} \mathrm{BKT130}$ or untreated control macrophages was collected at day 7 of monocyte cell culture and kept in $-20^{\circ} \mathrm{C}$ until use. Treatment with BKT130 took place at the day of macrophage polarization or at day 5 for the nonactivated macrophages (M0).

Adult C57BL6J mice, which were treated in accordance with the guidelines of the Association for Research in Vision and Ophthalmology (ARVO), were utilized for CSA experiments. Experiments were conducted with the approval of the institutional animal care ethics committee (see Ethical Approval). Mice were anesthetized with ketamine, checked for responses, and euthanized by cervical dislocation. The eyes were immediately enucleated and kept in ice-cold ECGS medium containing $1 / 100$ penicillin-streptomycin and $1 / 100$ glutamine before dissection. A choroid-sclera complex from the mice was gently dissected along with retinal pigment epithelium (RPE). The complex was cut into 5-6 $1 \mathrm{~mm}$ long pieces. Fragments were embedded in $30 \mu \mathrm{l}$ of growth factor-reduced Matrigel $^{\mathrm{TM}}$ (BD Biosciences, Cat. 354230) in 24-well plates. The thickness of the Matrigel ${ }^{\mathrm{TM}}$ was approximately $0.4 \mathrm{~mm}$. Plates were then incubated for 10 minutes in $37^{\circ} \mathrm{C}$, in a $5 \% \mathrm{CO}_{2}$ cell culture incubator without medium to solidify the Matrigel ${ }^{\mathrm{TM}}$. Medium $(250 \mu \mathrm{l})$ containing ECGM (C-22010, PromoCell, Germany), 2.5\% supplement mix (C-9215, PromoCell, Germany), 5\% FCS, 1/100 penicillin-streptomycin, and 1/100 glutamine was added to each well. $250 \mu \mathrm{l}$ of the macrophages' supernatant or $250 \mu \mathrm{l}$ of medium only was added to each well in duplicates. In addition, BKT130 was added directly to another group of CSA wells with the supernatant of untreated polarized M0 and
M1 macrophages from 5 other nvAMD patients (4 female, 1 male, mean age \pm SEM: $77.8 \pm 3.9$ years, range: $64-87$ ), to assess the effect of BKT130 directly on the supernatant without its potential effect on the macrophages' phenotype. Medium was changed every 3 days, and the cultures were fixed with $4 \%$ PFA after 8 days. Cultures were viewed with an inverted phase-contrast CKX41 Olympus microscope, and images were photographed with an Olympus DP70 digital camera (Olympus, Tokyo, Japan).

ImageJ software was used to quantify the sprouting area. The scale was set to convert pixels to $\mathrm{mm}^{2}$. Each image was converted to an 8-bit format to obtain a binary image. Sprouting area quantification and analysis were performed in duplicates for each sample.

2.6. Statistical Analysis. Data was processed using the biostatistical package InStat (GraphPad, San Diego, CA). $P<0.05$ was considered to indicate the statistical significance. Values over two standard deviations from the average were excluded from the statistical analysis. Appropriate statistical tests were applied according to the results of a normalcy test, sample distribution, and nature of the parameters.

\section{Results}

3.1. BKT130 Suppresses Laser-Induced Model of CNV (LI$C N V)$. The LI-CNV rat was utilized to evaluate the in vivo effect of BKT130 on CNV growth (Figure 2). The CNV area was measured 10 days after the induction of LI-CNV and commencement of intravitreal therapy in the rat eyes. BKT130 treatment ( $n=9$ eyes) was associated with a $36.8 \%$ reduction in the $\mathrm{CNV}$ area $\left[\right.$ mean area $\left(\mathrm{mm}^{2}\right) \pm \mathrm{SEM}$ ] as compared with control $(n=10)$ PBS-injected eyes $(0.036 \pm$ 0.005 vs. $0.057 \pm 0.004$, respectively; $P=0.005$, ANOVA). Aflibercept treatment $(n=8)$ was associated with a $68.4 \%$ reduction of the $\mathrm{CNV}$ area as compared with controls $(0.018 \pm 0.001, P=0.0001)$. Injection of both aflibercept and BKT130 $(n=8)$ resulted in a $70.2 \%$ smaller $\mathrm{CNV}$ area $(0.017 \pm 0.001, P=0.0001)$. CNV was $50 \%$ smaller in aflibercept-treated eyes compared with BKT130-treated eyes $(P=0.0003)$ and $52.8 \%$ smaller in aflibercept+BKT130treated eyes $(P=0.0003)$.

3.2. BKT130 Inhibits Mononuclear Phagocyte Recruitment to a LI-CNV. Immunostaining for CD11b+ cells was performed on the photoreceptor side of the retina flat mounts to assess their recruitment to the LI-CNV (Figure 1). CD11b+ cells were found beneath the photoreceptors (between retinal and RPE cells) overlying the laser injury site (Figure 1(a)). Laser injury sites were of similar size across the experimental groups, while the number of CD11b+ cells was associated with the specific treatment provided (Figure 1(a)). The lowest $\mathrm{CD} 11 \mathrm{~b}+$ cell count (43\% reduction) was found in the aflibercept+BKT130 (number of laser-treated areas $=41$; mean cell count in each laser-treated area \pm SEM: $18.51 \pm$ 1.26) as compared with the control PBS-treated eyes $(n=24$ laser injury areas, mean cell count $=32.63 \pm 2.23$; $P<0.001$; ANOVA). A reduction in the number of CD11b+ cells was also found in BKT130-treated eyes $(n=19$, cell 

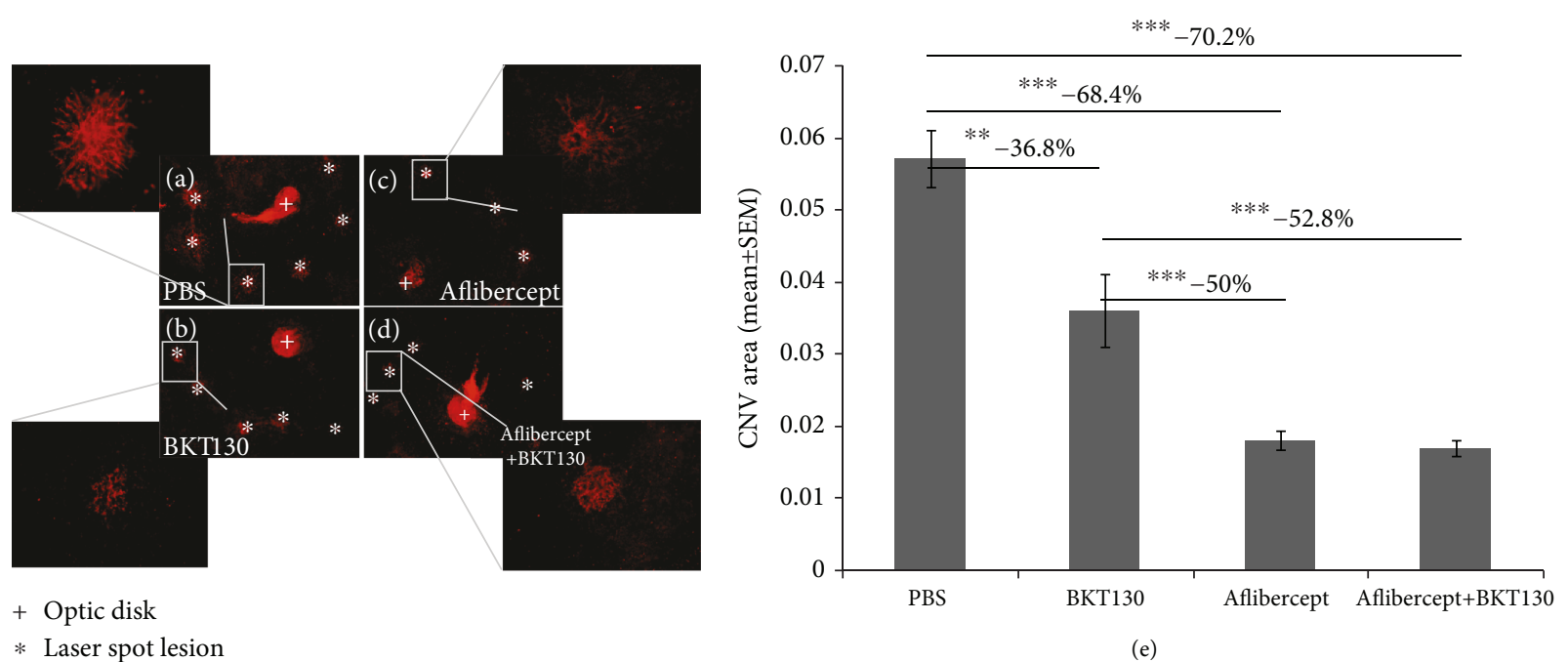

(e)

FIGURE 2: In vivo assessment of BKT130's effect in a rodent model of LI-CNV. BKT130 was injected intravitreally in a rat model of LI-CNV ( $n=9$ eyes). The eyes injected with PBS served as a negative control $(n=10)$, while intravitreal injections of aflibercept served as the positive control $(n=8)$. BKT130 was also injected with aflibercept to assess an additive effect $(n=8)$. CNV was identified and quantified using a fluorescent microscope in isolectin-stained RPE-choroid flat mounts (a-d). Each laser treated area was observed in a 20x lens and the whole flat mount in $4 \mathrm{x}$ lens. The CNV area was measured and compared between treatments and between PBS-injected eyes (e). The $Y$-axis presents the averaged $( \pm \mathrm{SEM}) \mathrm{CNV}$ area $\left(\mathrm{mm}^{2}\right)$ of treated and PBS-injected control eyes. ${ }^{* *} P<0.005$ and ${ }^{* * *} P<0.0005$.

count $=21.89 \pm 1.85 ; P<0.01 ;$ ANOVA $)$ and in aflibercepttreated eyes $(n=25,21.36 \pm 1.78 ; P<0.001$; ANOVA $)$ as compared to PBS-treated eyes. No difference in the mean cell number was found among BKT130, aflibercept, and combined aflibercept+BKT130-treated eyes.

\subsection{BKT130 Treatment Affects Gene Expression Profile in the} Eyes with LI-CNV. The mRNA expression profile of several genes evaluated with QPCR was associated with the specific treatments provided, as well as the tissue tested (retina and RPE-choroid; Figure 3).

Mean RPE-choroid CCR2 expression (RQ \pm SEM) was 2fold lower in the BKT130-treated eyes $(n=9$ eyes, $1.3 \pm 0.26)$ as compared with PBS $(n=9,2.56 \pm 0.37, P=0.02$, $t$-test) and 1.8-fold lower from aflibercept+BKT130-treated eyes $(n=9,2.35 \pm 0.37, P=0.05, t$-test) (Figure 3(a)). Multivariate analysis for CCR2 mRNA levels in the RPE-choroid across the four groups did not disclose a difference $(P=0.2$, Kruskal-Wallis test).

CCL5 mRNA levels in the RPE-choroid was 2-fold higher in BKT130-treated eyes $(0.9 \pm 0.14, P=0.03, t$-test $)$ and 3 fold higher in aflibercept-treated eyes $(n=9,1.37 \pm 0.36$, $P=0.04, t$-test), as compared to the eyes injected with PBS $(0.45 \pm 0.09$; Figure $3(\mathrm{~b}))$. Multivariate analysis for CCL5 mRNA levels across the four groups in the RPE-choroid did not disclose a difference ( $P=0.15$, Kruskal-Wallis test).

CCR2 and CCL5 expression in the retinal tissue was similar among the experimental groups.

TNF $\alpha$ expression was 2.25-fold lower in RPE-choroid $(0.8 \pm 0.17)$ and 2.7 -fold lower in retinal $(1.05 \pm 0.28)$ tissues of BKT130-treated eyes as compared to the PBS-treated eyes $(1.8 \pm 0.28, P=0.03$, Mann-Whitney test; $2.83 \pm 0.67, P=$ $0.05, t$-test, respectively) (Figures $3(\mathrm{c})-3(\mathrm{e})$ ). A multivariate test for retinal TNF $\alpha$ expression across the four groups con- firmed variable expression levels among the groups ( $P=0.04$, Kruskal-Wallis test). A multivariate test for RPEchoroid TNF $\alpha$ expression across the four groups did not disclose a difference $(P=0.1$, Kruskal-Wallis test), yet when we pooled the two groups that were treated with BKT130 (BKT130 and aflibercept+BKT130), the multivariate test for RPE-choroid TNF $\alpha$ expression across the three groups confirmed variable expression levels among the groups $(P=$ 0.04, Kruskal-Wallis test).

Retinal VEGFA expression was 1.8-fold lower in aflibercept-treated eyes $(0.32 \pm 0.03)$ as compared to PBStreated eyes $(0.59 \pm 0.05, P=0.0003, t$-test $)$. BKT130 treatment was associated with 3.2-fold reduced expression of $V E G F A$ in the RPE-choroid $(0.42 \pm 0.12)$ as compared to PBS-treated eyes $(1.34 \pm 0.28, P=0.02$, $t$-test $)$. The combination of aflibercept+BKT130 was associated with lower VEGFA expression by 1.25 -fold in retinal tissue $(0.47 \pm$ 0.04 ) and 2.6-fold reduced levels in RPE-choroid tissue $(0.52 \pm 0.12)$ as compared with the PBS-treated group $(0.59 \pm 0.05, P=0.05, t$-test; $1.34 \pm 0.28, P=0.01$, $t$-test, respectively) (Figures $3(\mathrm{~d})-3(\mathrm{f}))$. A multivariate test for $V E G F A$ expression across the four groups disclosed variable expression levels $(P=0.03$ in the retina and $P=0.004$ in the RPE-choroid, Kruskal-Wallis test).

A multivariate test for $C D 11 b$ expression in the retina across the four groups disclosed variable expression levels $(P=0.003$, Kruskal-Wallis test). Univariate analysis suggested that $C D 11 b$ expression in the retina was decreased by 14 -fold following BKT130 $(0.026 \pm 0.01)$ treatment and by 10 -fold following aflibercept treatment $(0.03 \pm 0.01)$ as compared with PBS- $(0.4 \pm 0.1)$ treated eyes $(P=0.02$, $t$-test; $P=0.02, t$-test, respectively) (Figure $3(\mathrm{~g})$ ). No change was measured in CD11b expression in the RPEchoroid tissue. 


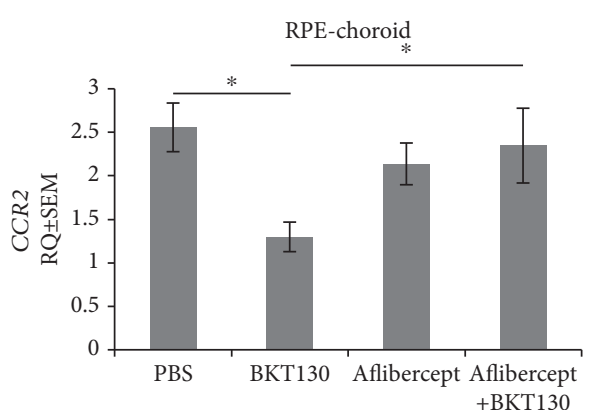

(a)

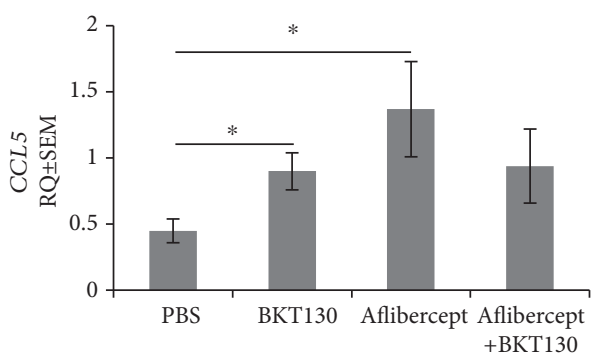

(b)

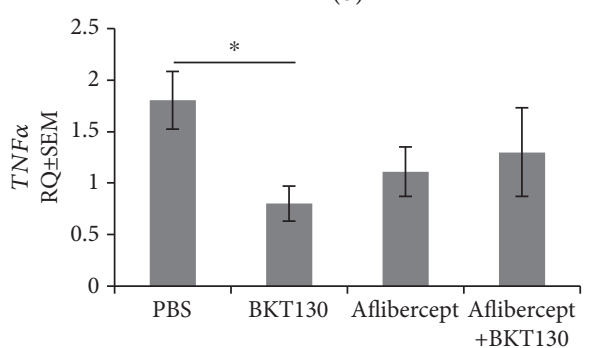

(c)

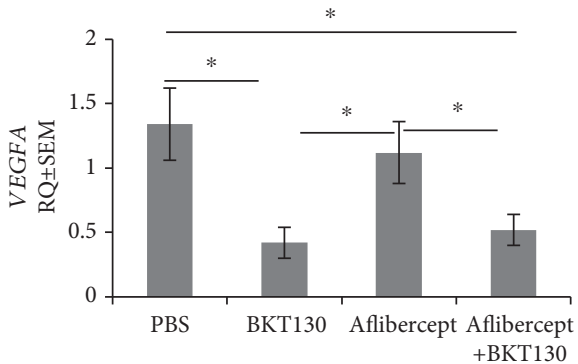

(d)

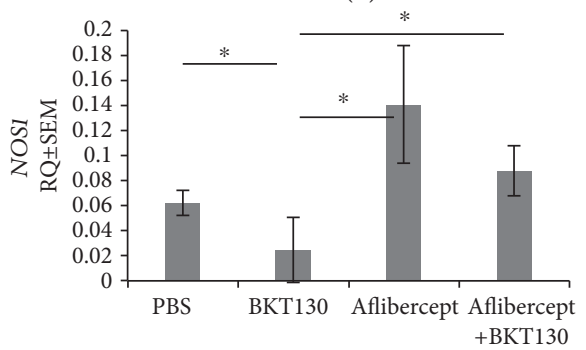

(i)

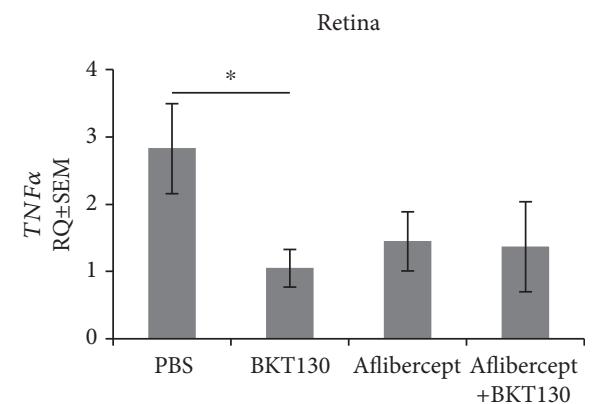

(e)

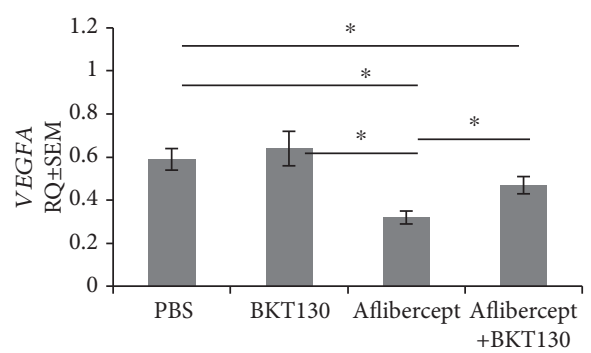

(f)

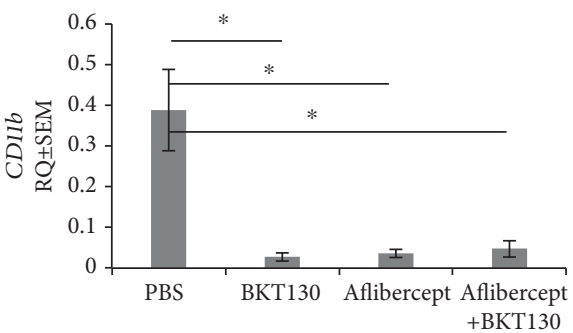

(g)

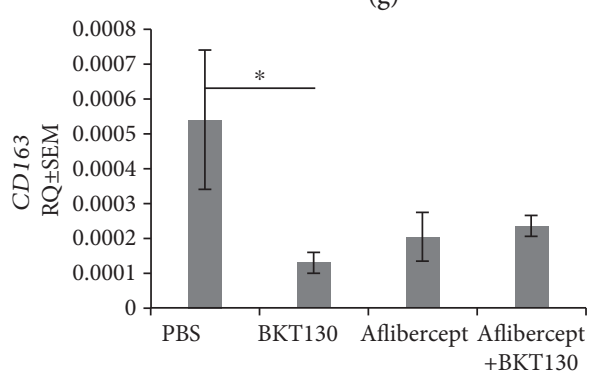

(h)

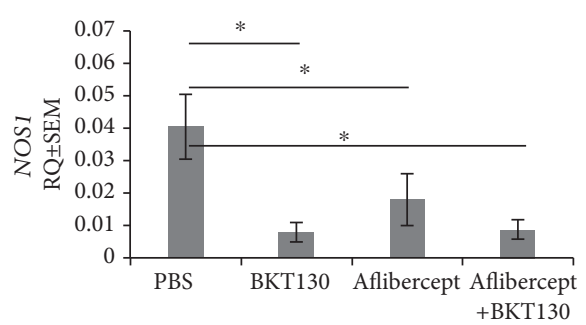

(j)

FIGURE 3: Gene expression profile of the retinas and RPE-choroid following treatment. mRNA expression levels of genes related to angiogenesis (VEGFA, IL1 $\beta$, and TNF $\alpha$ ), inflammation (CCL2, CCR2, CCL5, TNF, NAP-2, and MIP-2), mononuclear cell markers (NOS1, CD163, and CD11b), and macrophage recruitment (CCL2, CCR2, NAP-2, and MIP-2) were evaluated in the RPE-choroid (a-d) and in the retinas $(\mathrm{e}-\mathrm{h})$ of rats via QPCR $(n=9$ eyes in each group: PBS, BKT130, aflibercept, and BKT130+aflibercept). Presented are the genes that significantly changed after treatment. The $Y$-axis indicates RQ $\pm \mathrm{SEM}$. ${ }^{*} P<0.05$. 
A multivariate test for $C D 163$ expression in the retina across the four groups did not disclose a difference $(P=0.1$, Kruskal-Wallis test). Univariate analysis demonstrated that BKT130 treatment was associated with a 4.17-fold reduction of CD163 (an M2 macrophage biomarker) expression in the retina $(0.0005 \pm 0.0002$ and $0.0001 \pm 0.00003, P=0.03$, $t$-test, respectively) (Figure 3(h)). No change was measured in CD163 expression in the RPE-choroid tissue.

In univariate analysis, BKT130 treatment was associated with reduced NOS1 (an M1 macrophage biomarker) expression in the RPE-choroid and in the retina, respectively (RPEchoroid: BKT130-0.02 \pm 0.02 , PBS $-0.06 \pm 0.01, P=0.05$; retina: BKT130-0.008 \pm 0.003 , PBS $-0.04 \pm 0.01, P=0.01$, $t$-test). Aflibercept monotherapy was not associated with altered NOS1 expression in the choroid or the retina tissues. The combination therapy of aflibercept+BKT130 was associated with reduced NOS1 levels in the retina (aflibercept +BKT130: $0.008 \pm 0.003$, PBS: $0.04 \pm 0.01, P=0.02, t$-test), but not in the RPE-choroid. A multivariate test for NOS1 expression in the retina and in the RPE-choroid across the four groups disclosed variable expression levels $(P=0.04$ for both tissues, Kruskal-Wallis test) (Figures 3(i) and 3(j)).

No difference in the expression levels of MRC1, CCL2, IL1 $\beta, N A P-2$, and MIP-2 in the retina or in the RPEchoroid was identified across the treatment groups (data not shown).

3.4. BKT130 Does Not Affect Macrophages' Proangiogenic Phenotype or Function. An ex vivo CSA was conducted to evaluate the effect of BKT130 on macrophage's proangiogenic phenotype and the function of the secreted proteins. No difference in the sprouting area was detected among wells treated with the supernatant of macrophages that were incubated with or without BKT130 $(n=6$ in each group, mean of CSA area in $\mathrm{mm}^{2} \pm$ SEM, M0: untreated $1.72 \mathrm{~mm}^{2}$ \pm 0.32 vs. treated $1.64 \mathrm{~mm}^{2} \pm 0.32, P=0.7 ; \mathrm{M1}$ : untreated $2.2 \mathrm{~mm}^{2} \pm 0.34$ vs. treated $2.2 \pm 0.35, P=0.4 ; \mathrm{M} 2$ : untreated $1.62 \mathrm{~mm}^{2} \pm 0.34$ vs. treated $1.54 \mathrm{~mm}^{2} \pm 0.25, P=0.6$; paired $t$-test). In addition, the sprouting area was not affected by the addition of BKT130 to the CSA wells treated with macrophage's culture media in each macrophage subtype tested (mean of CSA area in $\mathrm{mm}^{2} \pm$ SEM, M0: without BKT130 $1.4 \mathrm{~mm}^{2} \pm 0.6$ vs. with BKT130 $1.12 \mathrm{~mm}^{2} \pm 0.6$, $P=0.8 ;$ M1: without BKT130 $2.2 \mathrm{~mm}^{2} \pm 0.9$ vs. with BKT130 $1.36 \mathrm{~mm}^{2} \pm 0.5, P=0.5$, paired $t$-test) (Figure 4).

\section{Discussion}

We describe the effect of a novel promiscuous chemokine antagonist (BKT130) in the rat model of LI-CNV. Application of this compound via the intravitreal route was associated with a reduction in the recruitment of $\mathrm{CD} 11 \mathrm{~b}+$ cells to the proximity of CNV lesions, a reduction of CNV size, and suppression of the expression of chemokines and cytokines, including the major monocyte receptor-CCR2-in the RPE-choroid tissue. Despite the fact that BKT130 inhibits chemokines which are expressed not only by the inflamed tissue but also by M1 and M2 macrophages, ex vivo treatment

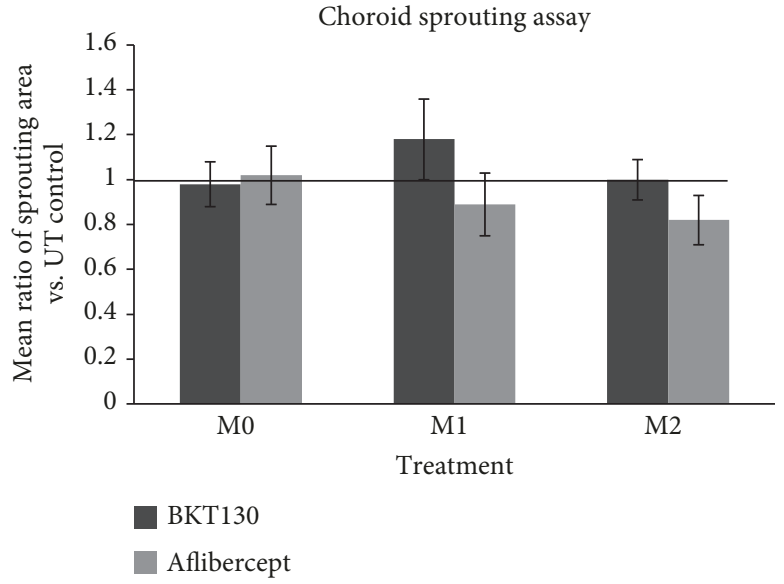

Figure 4: Choroid sprouting assay.

with BKT130 in CSA or treatment of cultured macrophages with BKT130 failed to suppress choroidal sprouting.

These data suggest that BKT130's favorable in vivo effect is mediated via perturbation of chemokine signaling and monocyte recruitment to the laser-injured area. Recently, it was suggested that microglia are resident macrophages of the retina that are derived from embryonic yolk sac progenitors during development, while nonresident bone marrowderived macrophages may be recruited into the retina from the vasculature in pathology [62]. Therefore, any additional $\mathrm{CD} 11 \mathrm{~b}+$ cells found in the retina are likely to represent recruited macrophages rather than resident microglia [63]. Macrophages were implicated in the pathogenesis of AMD based on multiple studies, among them are the presence of macrophages in the vicinity of AMD lesions [44, 45, 64, 65], proangiogenic human and rodent macrophages' effect in vitro and in the rodent model of LI-CNV $[18,22,66]$, and the reduced size of experimental CNV following inhibition of the CCR-CCL2 signaling pathway and monocyte recruitment $[30,67]$.

BKT130 suppresses LI-CNV via antagonizing multiple chemokines, thereby indirectly suppressing the expression of VEGF and other proinflammatory and proangiogenic cytokines. In the present study, anti-VEGF therapy was also associated with reduced macrophage recruitment, conceivably, through a PGF trap which inhibits subretinal phagocyte accumulation and other different mechanisms [68-70].

Macrophages may mediate CNV progression via cytokine production. TNF $\alpha$-expressing macrophages were previously detected in CNVs excised from AMD patients [71]. Our previous study showed that M1 macrophages from nvAMD, which had a proangiogenic effect in the rat model of LI-CNV, also produce considerable amounts of TNF $\alpha$ [22]. In addition, it has been previously suggested that macrophages secreting TNF $\alpha$ in CNV stimulate RPE expression of VEGF [71,72] and that TNF $\alpha$ increases the secretion of VEGF $\mathrm{A}$ and $\mathrm{C}$ and leads to the upregulation of VEGF expression by human RPE cells and choroidal fibroblasts $[73,74]$. Our current results showed approximately $60 \%$ reduction in TNF $\alpha$ expression in both retinal and RPEchoroidal tissues following BKT130 treatment. In addition, 
we found reduced expression of CD11b and NOS1 in both retinal and RPE-choroid tissues following BKT130 treatment which suggest reduced mononuclear phagocyte recruitment and reduced polarization towards the M1 macrophage phenotype. Interestingly, aflibercept, while suppressing macrophage recruitment as evident by reduced $C D 11 b$ expression in the retina, did not suppress TNF $\alpha$, NOS1, or CCR2 expression suggesting that it did not affect macrophage polarization or polarized macrophages' recruitment. Thus, our findings support a potential suppression of M1 macrophages' polarization by BKT 130 while aflibercept may potentially suppress the recruitment mononuclear cells but not polarization to the M1 phenotype and therefore may have a different mechanism of action on CNV growth. These results are in accordance with a recent report of higher expression of M1 markers in the RPE-choroid of a mouse following laserinduced $\mathrm{CNV}$ as compared to M2 macrophages' markers which were increased in the retina [75]. M1 macrophage activation and M1-dominant polarization profile of microglia were also recently described in the degenerative retina of rd1 mice [76].

VEGF immunoreactivity was previously found to be greater in inflammatory and active CNV and was found in the RPE to a greater extent than found in macrophages [77]. In addition to the RPE [78] and macrophages, at least six more retinal cell types have the capacity to produce and secrete VEGF including astrocytes [79], Müller cells [80], endothelial cells [81], microglia [77], pericytes [82], and ganglion cells [83]. BKT130 downregulated VEGFA expression in the RPE-choroid, but not in the retina. By contrast, aflibercept downregulated VEGFA expression in the retina and not in the RPE-choroid. Interestingly, combining aflibercept with BKT130 treatments caused downregulation of VEGFA expression both in retina and in RPE-choroid tissues. These results may reflect the variable mechanism of VEGF suppression associated with the two compounds and suggest a potential complementary effect of the combined therapy.

Caveats of the current study include the fact that LI-CNV in rat is a wound-healing reaction that follows an insult at the level of Bruch's membrane and relies heavily on inflammation $[18,19]$ and that it does not directly reflect nvAMD. In addition, because of the absence of a defined macula in rodents, this rodent model does not fully mimic the complexity of human pathology [84]. However, this model was proven to be suitable for testing the efficacy of new drugs through systemic or intraocular administration and has shown a predictive value for drug effects in patients with AMD, for example, with aflibercept $[85,86]$. In addition, while we observed a trend towards enhanced suppression of $\mathrm{CNV}$ in the combination arm of aflibercept+BKT130, this arm did not show a smaller CNV size as compared with aflibercept monotherapy. Yet in the LI-CNV model, application of aflibercept essentially eliminated the neovascular tufts, thereby, resulting in a ceiling effect that does not allow for a functional effect of the combined therapy to be apparent. Such complete elimination of the CNV lesion is not usually achieved in nvAMD following anti-VEGF therapy. Thus, in the human pathology, there is a need for supplementing the effect of available therapies. Finally, the lower injection vol- ume used in the aflibercept monotherapy group as compared to other groups may theoretically interact with $\mathrm{CNV}$ size. Yet our control group was injected with $4 \mu \mathrm{l}$ of PBS, similar to the BKT130 group which was the main focus of this research. Furthermore, the highest injection volume was used in the $1 \mu \mathrm{l}+4 \mu \mathrm{l}$ of the BKT130+aflibercept group, and this group yielded suppression of CNV.

\section{Conclusion}

Intravitreal delivery of a promiscuous chemokine antagonist, BKT130, inhibited the recruitment of monocytes to the laser injury area, reduced CNV area in the LI-CNV rat model, and decreased expression of VEGFA and CCR2 in RPE-choroid and TNF $\alpha$ in both RPE-choroid and retinal tissues. Reduction in TNF $\alpha$ and NOS1 with BKT130 but not with aflibercept might suggest a different macrophage subtype inhibition and therefore an additional effect on different patients. Additionally, a combination therapy with BKT130 and anti-VEGF had an additive effect on VEGFA expression in the eyes of rats with LI-CNV. Future studies should evaluate if perturbation of chemokine signaling may serve as a novel therapeutic option in nvAMD to supplement anti-VEGF therapy.

\section{Abbreviations}

AMD: Age-related macular degeneration

nvAMD: Neovascular stage of AMD

RPE: Retinal pigment epithelium

CNV: Choroidal neovascularization

LI-CNV: Laser-induced model of choroidal neovascularization

CSA: Choroid sprouting assay

FCS: $\quad$ Fetal calf serum

VEGF: Vascular endothelial growth factor.

\section{Data Availability}

We are happy to provide any relevant raw data, to any scientist who wishes to use them. For more information, please contact the corresponding author at chowers@hadassah.org.il.

\section{Ethical Approval}

Ethical approval for all protocols involving animals was approved by the Authority for Biological and Biomedical Models (ABBM) and the University Ethics Committee for the Care and Use of Laboratory Animals in Hebrew University, which is certified by the Association for Assessment and Accreditation of Laboratory Animal Care (AAALAC) (ethical approval number MD-16-14796-3, NIH approval number OPRR-A01-5011). All researchers working with laboratory animals underwent approval by the ethics committee of the ABBM to allow them to ethically work with laboratory animals. All guidelines with regard to humane and ethical treatment of laboratory animals (from ARVO) were followed to the utmost, and all methods used in this 
study were carried out in accordance with the approved guidelines for the study.

\section{Disclosure}

The funders had no role in study design, data collection and analysis, decision to publish, or preparation of the manuscript.

\section{Conflicts of Interest}

SHL, LT, BR, MG, and IC declare no actual or potential conflicts of interest. MA and $\mathrm{OE}$ are employees of Biokine Therapeutics Ltd. AP serves as a consultant to Biokine Therapeutics Ltd. OE and AP are shareholders of Biokine Therapeutics Ltd.

\section{Authors' Contributions}

SHL conceived the study, carried out all experiments, analyzed the data, and wrote the manuscript. MA reviewed the manuscript. LT, BR, and MG participated in conducting the experiments. OE designed and produced the compound BKT130. AP conceived the study, obtained funding for the research, and coordinated it. IC conceived the study, analyzed the data, and participated in writing the manuscript. AP, MA, SHL, IC, and OE all participated in the study design, but none of them, except SHL, conducted the experiment or participated in the data analysis and/or results. All experiments are carried out in the lab of IC and by his students SHL, MG, BR, and LT. All authors read and approved the final manuscript.

\section{Acknowledgments}

This work was supported by a grant from Biokine Therapeutics Ltd. (\#6037411) (AP).

\section{Supplementary Materials}

Appendix Table 1: mRNA primers for QPCR. (Supplementary Materials)

\section{References}

[1] D. H. Anderson, M. J. Radeke, N. B. Gallo et al., "The pivotal role of the complement system in aging and age-related macular degeneration: hypothesis re-visited," Progress in Retinal and Eye Research, vol. 29, no. 2, pp. 95-112, 2010.

[2] The AMD Gene Consortium, "Seven new loci associated with age-related macular degeneration," Nature Genetics, vol. 45, no. 4, pp. 433-439, 2013.

[3] H. P. N. Scholl, P. C. Issa, M. Walier et al., "Systemic complement activation in age-related macular degeneration," PLoS One, vol. 3, no. 7, article e2593, 2008.

[4] B. E. K. Klein, C. E. Myers, K. P. Howard, and R. Klein, "Serum lipids and proliferative diabetic retinopathy and macular edema in persons with long-term type 1 diabetes mellitus," JAMA Ophthalmology, vol. 133, no. 5, pp. 503-510, 2015.

[5] R. Klein, S. M. Meuer, C. E. Myers et al., "Harmonizing the classification of age-related macular degeneration in the three-continent AMD consortium," Ophthalmic Epidemiology, vol. 21, no. 1, pp. 14-23, 2014.

[6] R. Klein, C. E. Myers, G. H. S. Buitendijk et al., "Lipids, lipid genes, and incident age-related macular degeneration: the three continent age-related macular degeneration consortium," American Journal of Ophthalmology, vol. 158, no. 3, pp. 513-524.e3, 2014.

[7] R. Klein, C. E. Myers, K. J. Cruickshanks et al., "Markers of inflammation, oxidative stress, and endothelial dysfunction and the 20-year cumulative incidence of early age-related macular degeneration: the Beaver Dam Eye Study," JAMA Ophthalmology, vol. 132, no. 4, pp. 446-455, 2014.

[8] R. Klein, C. E. Myers, and B. E. K. Klein, "Vasodilators, blood pressure-lowering medications, and age-related macular degeneration: the Beaver Dam Eye Study," Ophthalmology, vol. 121, no. 8, pp. 1604-1611, 2014.

[9] M. Grunin, S. Hagbi-Levi, and I. Chowers, "The role of monocytes and macrophages in age-related macular degeneration," Advances in Experimental Medicine and Biology, vol. 801, pp. 199-205, 2014.

[10] A. O. Edwards and G. Malek, "Molecular genetics of AMD and current animal models," Angiogenesis, vol. 10, no. 2, pp. 119132, 2007.

[11] J. L. Haines, M. A. Hauser, S. Schmidt et al., "Complement factor $\mathrm{H}$ variant increases the risk of age-related macular degeneration," Science, vol. 308, no. 5720, pp. 419-421, 2005.

[12] X. L. Tian, R. Kadaba, S. A. You et al., "Identification of an angiogenic factor that when mutated causes susceptibility to Klippel-Trenaunay syndrome," Nature, vol. 427, no. 6975, pp. 640-645, 2004.

[13] J. B. Maller, J. A. Fagerness, R. C. Reynolds, B. M. Neale, M. J. Daly, and J. M. Seddon, "Variation in complement factor 3 is associated with risk of age-related macular degeneration," Nature Genetics, vol. 39, no. 10, pp. 1200-1201, 2007.

[14] R. Zhang, K. G. Hadlock, H. Do et al., "Gene expression profiling in peripheral blood mononuclear cells from patients with sporadic amyotrophic lateral sclerosis (sALS)," Journal of Neuroimmunology, vol. 230, no. 1-2, pp. 114-123, 2011.

[15] S. H. Sarks, D. Van Driel, L. Maxwell, and M. Killingsworth, "Softening of drusen and subretinal neovascularization," Transactions of the Ophthalmological Societies of the United Kingdom, vol. 100, no. 3, pp. 414-422, 1980.

[16] D. H. Anderson, R. F. Mullins, G. S. Hageman, and L. V. Johnson, "A role for local inflammation in the formation of drusen in the aging eye," American Journal of Ophthalmology, vol. 134, no. 3, pp. 411-431, 2002.

[17] R. S. Apte, J. Richter, J. Herndon, and T. A. Ferguson, "Macrophages inhibit neovascularization in a murine model of agerelated macular degeneration," PLoS Medicine, vol. 3, no. 8, article e310, 2006.

[18] D. G. Espinosa-Heidmann, I. J. Suner, E. P. Hernandez, D. Monroy, K. G. Csaky, and S. W. Cousins, "Macrophage depletion diminishes lesion size and severity in experimental choroidal neovascularization," Investigative Ophthalmology \& Visual Science, vol. 44, no. 8, pp. 3586-3592, 2003.

[19] E. Sakurai, A. Anand, B. K. Ambati, N. van Rooijen, and J. Ambati, "Macrophage depletion inhibits experimental choroidal neovascularization," Investigative Ophthalmology \& Visual Science, vol. 44, no. 8, pp. 3578-3585, 2003.

[20] K. Kataoka, K. M. Nishiguchi, H. Kaneko, N. van Rooijen, S. Kachi, and H. Terasaki, "The roles of vitreal macrophages 
and circulating leukocytes in retinal neovascularization," Investigative Ophthalmology \& Visual Science, vol. 52, no. 3, pp. 1431-1438, 2011.

[21] M. J. Robertson, L. P. Erwig, J. Liversidge, J. V. Forrester, A. J. Rees, and A. D. Dick, "Retinal microenvironment controls resident and infiltrating macrophage function during uveoretinitis," Investigative Ophthalmology \& Visual Science, vol. 43, no. 7, pp. 2250-2257, 2002.

[22] S. Hagbi-Levi, M. Grunin, T. Jaouni et al., "Proangiogenic characteristics of activated macrophages from patients with age-related macular degeneration," Neurobiology of Aging, vol. 51, pp. 71-82, 2017.

[23] F. Cruz-Guilloty, A. M. Saeed, S. Duffort et al., "T cells and macrophages responding to oxidative damage cooperate in pathogenesis of a mouse model of age-related macular degeneration," PLoS One, vol. 9, no. 2, article e88201, 2014.

[24] A. Caicedo, D. G. Espinosa-Heidmann, Y. Pina, E. P. Hernandez, and S. W. Cousins, "Blood-derived macrophages infiltrate the retina and activate Muller glial cells under experimental choroidal neovascularization," Experimental Eye Research, vol. 81, no. 1, pp. 38-47, 2005.

[25] C. Faber, A. Singh, M. Krüger Falk, H. B. Juel, T. L. Sørensen, and M. H. Nissen, "Age-related macular degeneration is associated with increased proportion of $\mathrm{CD}^{2} 6^{+} \mathrm{T}$ cells in peripheral blood," Ophthalmology, vol. 120, no. 11, pp. 2310-2316, 2013.

[26] A. Mantovani, A. Sica, and M. Locati, "Macrophage polarization comes of age," Immunity, vol. 23, no. 4, pp. 344-346, 2005.

[27] R. S. Apte, "Regulation of angiogenesis by macrophages," Advances in Experimental Medicine and Biology, vol. 664, pp. 15-19, 2010.

[28] S. Zandi, S. Nakao, K. H. Chun et al., "ROCK-isoform-specific polarization of macrophages associated with age-related macular degeneration," Cell Reports, vol. 10, no. 7, pp. 11731186, 2015.

[29] A. Sene, A. A. Khan, D. Cox et al., "Impaired cholesterol efflux in senescent macrophages promotes age-related macular degeneration," Cell Metabolism, vol. 17, no. 4, pp. 549-561, 2013.

[30] C. Tsutsumi, K. H. Sonoda, K. Egashira et al., "The critical role of ocular-infiltrating macrophages in the development of choroidal neovascularization," Journal of Leukocyte Biology, vol. 74, no. 1, pp. 25-32, 2003.

[31] L. Li, P. Heiduschka, A. F. Alex, D. Niekämper, and N. Eter, "Behaviour of CD11b-positive cells in an animal model of laser-induced choroidal neovascularisation," Ophthalmologica, vol. 237, no. 1, pp. 29-41, 2017.

[32] C. N. Nagineni, V. K. Kommineni, N. Ganjbaksh, K. K. Nagineni, J. J. Hooks, and B. Detrick, "Inflammatory cytokines induce expression of chemokines by human retinal cells: role in chemokine receptor mediated age-related macular degeneration," Aging and Disease, vol. 6, no. 6, pp. 444-455, 2015.

[33] Y. F. Feng, H. Guo, F. Yuan, and M. Q. Shen, "Lipopolysaccharide promotes choroidal neovascularization by up-regulation of CXCR4 and CXCR7 expression in choroid endothelial cell," PLoS ONE, vol. 10, no. 8, article e0136175, 2015.

[34] M. Rutar, R. Natoli, R. X. Chia, K. Valter, and J. M. Provis, "Chemokine-mediated inflammation in the degenerating retina is coordinated by Müller cells, activated microglia, and retinal pigment epithelium," Journal of Neuroinflammation, vol. 12, no. 1, p. 8, 2015.
[35] M. Rutar, R. Natoli, and J. M. Provis, "Small interfering RNAmediated suppression of Ccl2 in Müller cells attenuates microglial recruitment and photoreceptor death following retinal degeneration," Journal of Neuroinflammation, vol. 9, no. 1, p. 221, 2012.

[36] F. M. Mo, A. D. Proia, W. H. Johnson, D. Cyr, and K. Lashkari, "Interferon $\gamma$-inducible protein-10 (IP-10) and eotaxin as biomarkers in age-related macular degeneration," Investigative Ophthalmology \& Visual Science, vol. 51, no. 8, pp. 42264236, 2010.

[37] V. Torraca, C. Cui, R. Boland et al., "The CXCR3-CXCL11 signaling axis mediates macrophage recruitment and dissemination of mycobacterial infection," Disease Models \& Mechanisms, vol. 8, no. 3, pp. 253-269, 2015.

[38] D. Petrovic-Djergovic, M. Popovic, S. Chittiprol, H. Cortado, R. F. Ransom, and S. Partida-Sánchez, "CXCL10 induces the recruitment of monocyte-derived macrophages into kidney, which aggravate puromycin aminonucleoside nephrosis," Clinical and Experimental Immunology, vol. 180, no. 2, pp. 305-315, 2015.

[39] A. M. Newman, N. B. Gallo, L. S. Hancox et al., "Systems-level analysis of age-related macular degeneration reveals global biomarkers and phenotype-specific functional networks," Genome Medicine, vol. 4, no. 2, p. 16, 2012.

[40] S. Fujimura, H. Takahashi, K. Yuda et al., "Angiostatic effect of CXCR3 expressed on choroidal neovascularization," Investigative Ophthalmology \& Visual Science, vol. 53, no. 4, pp. 19992006, 2012.

[41] M. Grunin, T. Burstyn-Cohen, S. Hagbi-Levi, A. Peled, and I. Chowers, "Chemokine receptor expression in peripheral blood monocytes from patients with neovascular age-related macular degeneration," Investigative Opthalmology \& Visual Science, vol. 53, no. 9, article 5292, 2012.

[42] M. Kramer, M. Hasanreisoglu, A. Feldman et al., "Monocyte chemoattractant protein-1 in the aqueous humour of patients with age-related macular degeneration," Clinical \& Experimental Ophthalmology, vol. 40, no. 6, pp. 617-625, 2012.

[43] J. B. Jonas, Y. Tao, M. Neumaier, and P. Findeisen, "Cytokine concentration in aqueous humour of eyes with exudative agerelated macular degeneration," Acta Ophthalmologica, vol. 90, no. 5, pp. e381-e388, 2012.

[44] P. L. Penfold, M. C. Killingsworth, and S. H. Sarks, "Senile macular degeneration: the involvement of immunocompetent cells," Graefe's Archive for Clinical and Experimental Ophthalmology, vol. 223, no. 2, pp. 69-76, 1985.

[45] M. C. Killingsworth, J. P. Sarks, and S. H. Sarks, "Macrophages related to Bruch's membrane in age-related macular degeneration," Eye, vol. 4, no. 4, pp. 613-621, 1990.

[46] S. Cherepanoff, P. McMenamin, M. C. Gillies, E. Kettle, and S. H. Sarks, "Bruch's membrane and choroidal macrophages in early and advanced age-related macular degeneration," The British Journal of Ophthalmology, vol. 94, no. 7, pp. 918925,2010

[47] X. Cao, D. Shen, M. M. Patel et al., "Macrophage polarization in the maculae of age-related macular degeneration: a pilot study," Pathology International, vol. 61, no. 9, pp. 528-535, 2011.

[48] P. L. Penfold, J. G. Wong, J. Gyory, and F. A. Billson, "Effects of triamcinolone acetonide on microglial morphology and quantitative expression of MHC-II in exudative age-related macular degeneration," Clinical \& Experimental Ophthalmology, vol. 29, no. 3, pp. 188-192, 2001. 
[49] F. Sennlaub, C. Auvynet, B. Calippe et al., "CCR2(+) monocytes infiltrate atrophic lesions in age-related macular disease and mediate photoreceptor degeneration in experimental subretinal inflammation in Cx3cr1 deficient mice," EMBO Molecular Medicine, vol. 5, no. 11, pp. 1775-1793, 2013.

[50] S. J. Allen, S. E. Crown, and T. M. Handel, "Chemokine: receptor structure, interactions, and antagonism," Annual Review of Immunology, vol. 25, no. 1, pp. 787-820, 2007.

[51] H. Kohno, T. Maeda, L. Perusek, E. Pearlman, and A. Maeda, "CCL3 production by microglial cells modulates disease severity in murine models of retinal degeneration," Journal of Immunology, vol. 192, no. 8, pp. 3816-3827, 2014.

[52] S. Joly, M. Francke, E. Ulbricht et al., "Cooperative phagocytes: resident microglia and bone marrow immigrants remove dead photoreceptors in retinal lesions," The American Journal of Pathology, vol. 174, no. 6, pp. 2310-2323, 2009.

[53] N. Fernando, R. Natoli, K. Valter, J. Provis, and M. Rutar, “The broad-spectrum chemokine inhibitor NR58-3.14.3 modulates macrophage-mediated inflammation in the diseased retina," Journal of Neuroinflammation, vol. 13, no. 1, p. 47, 2016.

[54] M. Abraham, H. Wald, D. Vaizel-Ohayon et al., "Development of novel promiscuous anti-chemokine peptibodies for treating autoimmunity and inflammation," Frontiers in Immunology, vol. 8, article 1432, 2017.

[55] T. Tobe, N. Okamoto, M. A. Vinores et al., "Evolution of neovascularization in mice with overexpression of vascular endothelial growth factor in photoreceptors," Investigative Ophthalmology \& Visual Science, vol. 39, no. 1, pp. 180-188, 1998.

[56] T. J. Collins, “ImageJ for microscopy," BioTechniques, vol. 43, 1 Supplement, pp. S25-S30, 2007.

[57] K. J. Livak and T. D. Schmittgen, "Analysis of relative gene expression data using real-time quantitative PCR and the 2 ${ }_{-}^{\Delta \Delta \mathrm{C}}{ }_{\mathrm{T}}$ method," Methods, vol. 25, no. 4, pp. 402-408, 2001.

[58] The Age-Related Eye Disease Study Research Group, “The Age-Related Eye Disease Study (AREDS): design implications AREDS report no. 1," Controlled Clinical Trials, vol. 20, no. 6, pp. 573-600, 1999.

[59] A. Mantovani, S. Sozzani, M. Locati, P. Allavena, and A. Sica, "Macrophage polarization: tumor-associated macrophages as a paradigm for polarized M2 mononuclear phagocytes," Trends in Immunology, vol. 23, no. 11, pp. 549-555, 2002.

[60] F. O. Martinez, L. Helming, and S. Gordon, "Alternative activation of macrophages: an immunologic functional perspective," Annual Review of Immunology, vol. 27, no. 1, pp. 451483, 2009.

[61] Z. Shao, M. Friedlander, C. G. Hurst et al., "Correction: choroid sprouting assay: an ex vivo model of microvascular angiogenesis," PLoS One, vol. 8, no. 8, article e69552, 2013.

[62] E. G. O'Koren, R. Mathew, and D. R. Saban, “Fate mapping reveals that microglia and recruited monocyte-derived macrophages are definitively distinguishable by phenotype in the retina," Scientific Reports, vol. 6, no. 1, article 20636, 2016.

[63] S. M. Hector and T. L. Sørensen, "Circulating monocytes and B-lymphocytes in neovascular age-related macular degeneration," Clinical Ophthalmology, vol. 11, pp. 179-184, 2017.

[64] P. Penfold, M. Killingsworth, and S. Sarks, "An ultrastructural study of the role of leucocytes and fibroblasts in the breakdown of Bruch's membrane," Australian Journal of Ophthalmology, vol. 12, no. 1, pp. 23-31, 1984.
[65] J. T. Rosenbaum, L. O'rourke, G. Davies, C. Wenger, L. David, and J. E. Robertson, "Retinal pigment epithelial cells secrete substances that are chemotactic for monocytes," Current Eye Research, vol. 6, no. 6, pp. 793-800, 1987.

[66] D. G. Espinosa-Heidmann, A. Caicedo, E. P. Hernandez, K. G. Csaky, and S. W. Cousins, "Bone marrow-derived progenitor cells contribute to experimental choroidal neovascularization," Investigative Ophthalmology \& Visual Science, vol. 44, no. 11, pp. 4914-4919, 2003.

[67] P. Xie, M. Kamei, M. Suzuki et al., "Suppression and regression of choroidal neovascularization in mice by a novel CCR2 antagonist, INCB3344," PLoS One, vol. 6, no. 12, article e28933, 2011.

[68] M. Grunewald, I. Avraham, Y. Dor et al., "VEGF-induced adult neovascularization: recruitment, retention, and role of accessory cells," Cell, vol. 124, no. 1, pp. 175-189, 2006.

[69] E. C. Breen, "VEGF in biological control," Journal of Cellular Biochemistry, vol. 102, no. 6, pp. 1358-1367, 2007.

[70] S. Crespo-Garcia, C. Corkhill, C. Roubeix et al., "Inhibition of placenta growth factor reduces subretinal mononuclear phagocyte accumulation in choroidal neovascularization," Investigative Ophthalmology and Visual Science, vol. 58, no. 12, pp. 4997-5006, 2017.

[71] H. Oh, H. Takagi, C. Takagi et al., "The potential angiogenic role of macrophages in the formation of choroidal neovascular membranes," Investigative Ophthalmology \& Visual Science, vol. 40, no. 9, pp. 1891-1898, 1999.

[72] A. Otani, H. Takagi, H. Oh, S. Koyama, M. Matsumura, and Y. Honda, "Expressions of angiopoietins and Tie2 in human choroidal neovascular membranes," Investigative Ophthalmology and Visual Science, vol. 40, no. 9, pp. 1912-1920, 1999.

[73] C. N. Nagineni, V. K. Kommineni, A. William, B. Detrick, and J. J. Hooks, "Regulation of VEGF expression in human retinal cells by cytokines: implications for the role of inflammation in age-related macular degeneration," Journal of Cellular Physiology, vol. 227, no. 1, pp. 116-126, 2012.

[74] H. Wang, X. Han, E. S. Wittchen, and M. E. Hartnett, “TNF- $\alpha$ mediates choroidal neovascularization by upregulating VEGF expression in RPE through ROS-dependent $\beta$-catenin activation," Molecular Vision, vol. 22, pp. 116-128, 2016.

[75] Y. Zhou, S. Yoshida, Y. Kubo et al., "Different distributions of M1 and M2 macrophages in a mouse model of laser-induced choroidal neovascularization," Molecular Medicine Reports, vol. 15, no. 6, pp. 3949-3956, 2017.

[76] T. Zhou, Z. Huang, X. Sun et al., "Microglia polarization with M1/M2 phenotype changes in rd1 mouse model of retinal degeneration," Frontiers in Neuroanatomy, vol. 11, 2017.

[77] H. E. Grossniklaus, J. X. Ling, T. M. Wallace et al., "Macrophage and retinal pigment epithelium expression of angiogenic cytokines in choroidal neovascularization," Molecular Vision, vol. 8, pp. 119-126, 2002.

[78] J. W. Miller, "Vascular endothelial growth factor and ocular neovascularization," The American Journal of Pathology, vol. 151, no. 1, pp. 13-23, 1997.

[79] J. Stone, A. Itin, T. Alon et al., "Development of retinal vasculature is mediated by hypoxia-induced vascular endothelial growth factor (VEGF) expression by neuroglia," The Journal of Neuroscience, vol. 15, no. 7, pp. 4738-4747, 1995.

[80] S. G. Robbins, J. R. Conaway, B. L. Ford, K. A. Roberto, and J. S. Penn, "Detection of vascular endothelial growth factor (VEGF) protein in vascular and non-vascular cells of the 
normal and oxygen-injured rat retina," Growth Factors, vol. 14, no. 4, pp. 229-241, 1997.

[81] L. P. Aiello, R. L. Avery, P. G. Arrigg et al., "Vascular endothelial growth factor in ocular fluid of patients with diabetic retinopathy and other retinal disorders," The New England Journal of Medicine, vol. 331, no. 22, pp. 1480-1487, 1994.

[82] D. C. Darland, L. J. Massingham, S. R. Smith, E. Piek, M. SaintGeniez, and P. A. D'Amore, "Pericyte production of cellassociated VEGF is differentiation-dependent and is associated with endothelial survival," Developmental Biology, vol. 264, no. 1, pp. 275-288, 2003.

[83] H. Ida, T. Tobe, H. Nambu, M. Matsumura, M. Uyama, and P. A. Campochiaro, "RPE cells modulate subretinal neovascularization, but do not cause regression in mice with sustained expression of VEGF," Investigative Ophthalmology and Visual Science, vol. 44, no. 12, pp. 5430-5437, 2003.

[84] J. W. Miller, “Age-related macular degeneration revisited piecing the puzzle: the LXIX Edward Jackson memorial lecture," American Journal of Ophthalmology, vol. 155, no. 1, pp. 1-35.e13, 2013.

[85] J. S. Heier, D. M. Brown, V. Chong et al., "Intravitreal aflibercept (VEGF trap-eye) in wet age-related macular degeneration," Ophthalmology, vol. 119, no. 12, pp. 2537-2548, 2012.

[86] Y. Y. Saishin, Y. Y. Saishin, K. Takahashi et al., "VEGFTRAPR1R2 suppresses choroidal neovascularization and VEGF-induced breakdown of the blood-retinal barrier," Journal of Cellular Physiology, vol. 195, no. 2, pp. 241-248, 2003. 


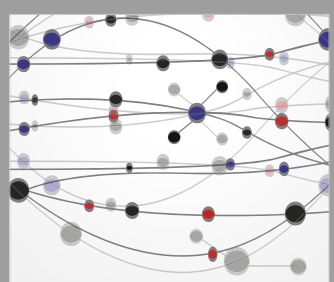

The Scientific World Journal
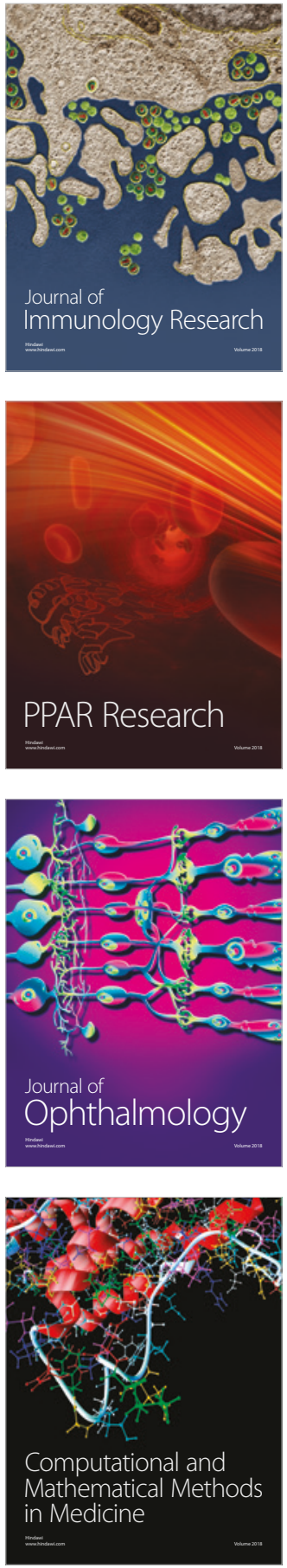

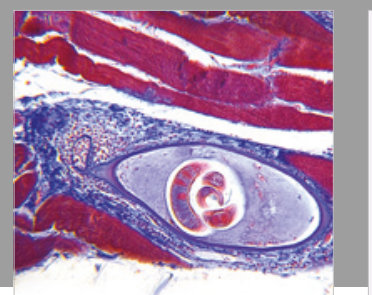

Gastroenterology Research and Practice

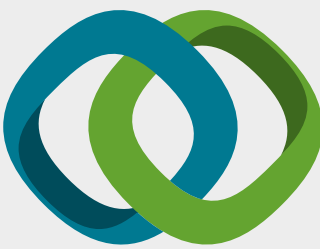

\section{Hindawi}

Submit your manuscripts at

www.hindawi.com
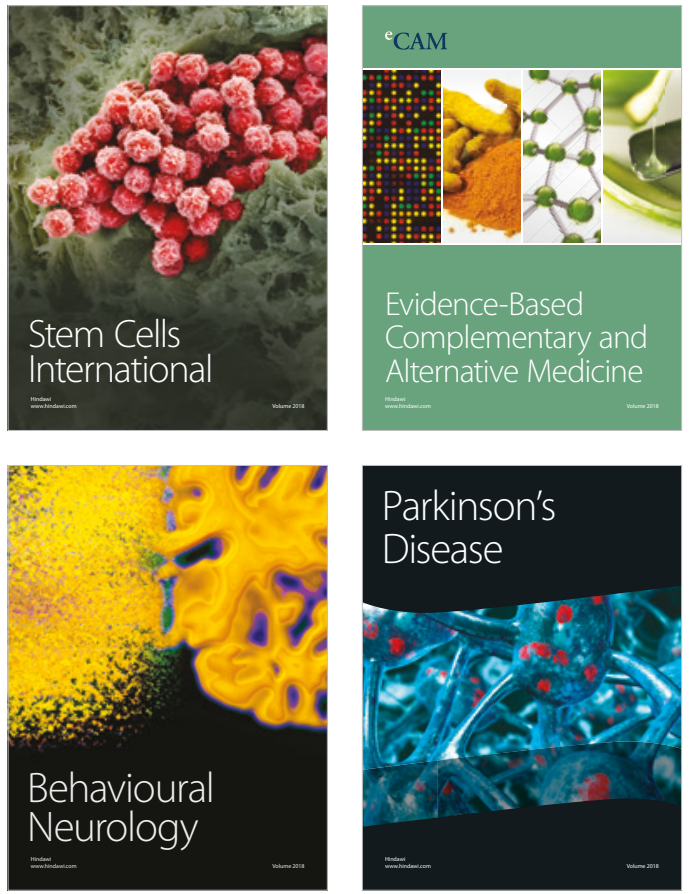

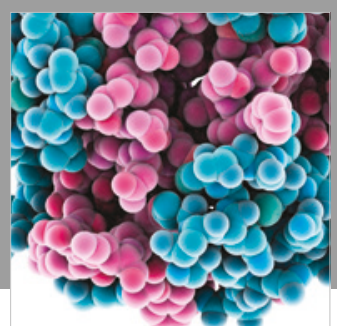

ournal of

Diabetes Research

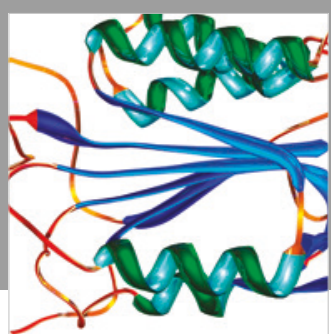

Disease Markers
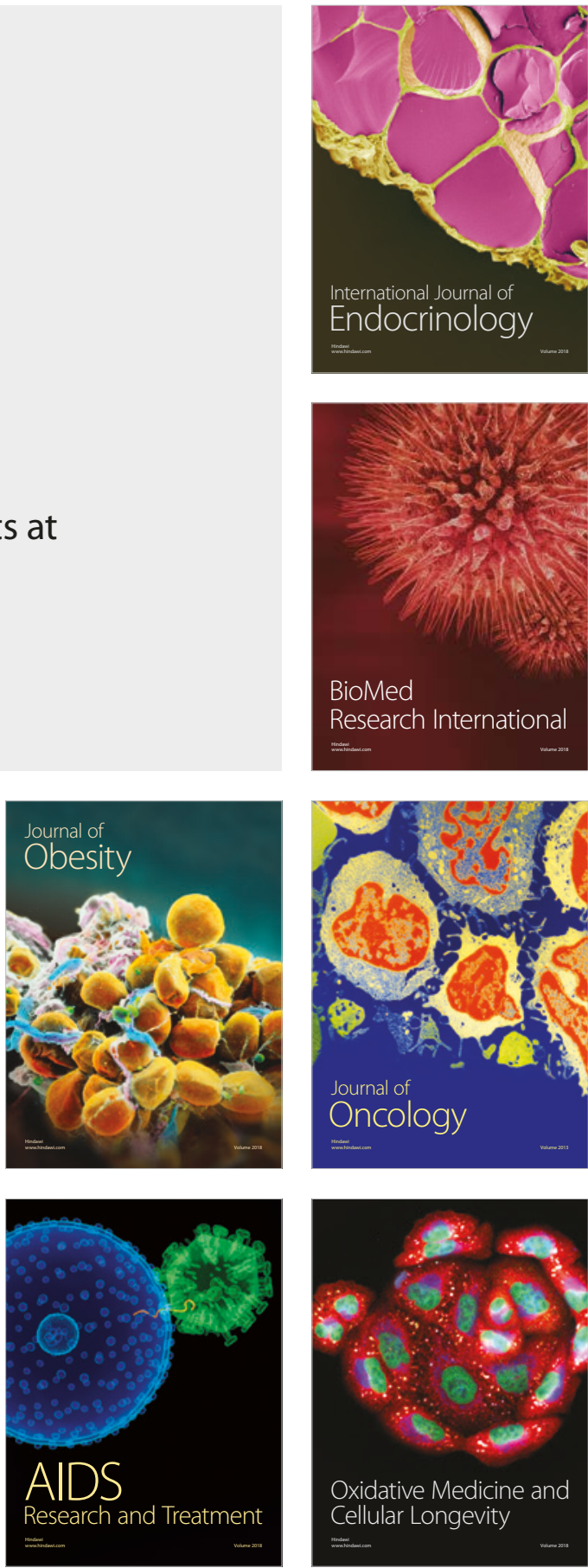